\title{
Crystal growth kinetics of calcite and its comparison with readily soluble salts
}

\author{
Clifford Y. Tai *, Hsiao-Ping Hsu \\ Department of Chemical Engineering, National Taiwan University, Roosevelt Road, Taipei 10617, Taiwan
}

\begin{abstract}
The crystal growth kinetics of calcite was investigated in batch crystallizers of stirred tank and fluidized-bed type, which were maintained at a constant $\mathrm{pH}$ by an autotitrator. Rhombohedron seed crystals were prepared using different techniques, then the growth experiments were conducted in the metastable region, which was explored as part of this research, to suppress nucleation. The crystal growth rates were evaluated from the consumption rates of sodium carbonate and calcium ion for the stirred-tank and fluidized-bed experiments, respectively. Several operating variables were investigated and the crystal growth rate data of constant $\mathrm{pH}$ and ionic strength were analyzed by the two-step crystal growth model. The mass-transfer and surface-reaction coefficients were thus obtained and used to explain the growth behaviors of calcite crystals. Then, the crystal growth kinetics of this sparingly soluble salt was compared with that of readily soluble salts. Although the effects of $\mathrm{pH}$ and ionic strength on crystal growth were not reported for the readily soluble systems, these effects were significant to calcite growth. (c) 2001 Elsevier Science B.V. All rights reserved.
\end{abstract}

Keywords: Calcite; pH-stat apparatus; Crystal growth kinetics; Stirred tank; Fluidized bed

\section{Introduction}

Crystal growth from solution of sparingly soluble system is rarely studied as compared with that of readily soluble system, especially for the growth of large seed crystals. Usually, fine crystals of sparingly soluble salts are generated by spontaneous nucleation via rapid mixing and reaction of two solutions, and they have no chance to grow. Thus, the study on crystal growth of large seed crystal of sparingly soluble salts had been ignored until the pellet reactor was applied to the field of environmental protection. A pellet reactor is a reactive, fluidized-bed, growth-type crystallizer used for water softening, fluoride and phosphate removal, and heavy metal recovery. The water feed and chemical reagents required to cause deposition are fed to the reactor containing suspended seeds [1,2]. The materials grown on the seed are mostly sparingly soluble salts. For example, the undesired species in water softening of drinking water is calcium ion, which reacts with the added carbonate to form calcium carbonate and

\footnotetext{
* Corresponding author. Fax: +886-2-2362-0832.

E-mail address: d3504007@ms.cc.ntu.edu.tw (C.Y. Tai).
}

then grow on the seeds [3]. The seeds are later removed from the reactor after they exceed a certain size, say, several hundred micrometers ready for solid-liquid separation.

To design a pellet reactor, the metastable region and the crystal growth rate should be known. The former gives the concentration zone where the reactor is operative, i.e. crystals grow without nucleation, and the latter determines the reactor size. The concept of metastable region for sparingly soluble system is illustrated by Söhnel and Garside [4]. The metastable region is present in a precipitation diagram by plotting $\mathrm{p} A$ versus $\mathrm{p} B$, where $A$ and $B$ are the concentrations of ion species and $\mathrm{p} A$ represents $-\log A$. The metastable region of sparingly soluble system is available for only a few systems, perhaps $\mathrm{CaCl}_{2}-\mathrm{Na}_{3} \mathrm{PO}_{4}-\mathrm{H}_{2} \mathrm{O}$ [4] and $\mathrm{CaSO}_{4} \cdot 2 \mathrm{H}_{2} \mathrm{O}-\mathrm{H}_{2} \mathrm{O}$ [5] are the two systems that can be found, and the latter system is plotted on an ionic product-temperature diagram instead of the precipitation diagram. In the study on crystal growth of sparingly soluble salts using seeded technique, the seed size is usually less than $10 \mu \mathrm{m}$ [6-8] because large crystals of sparingly soluble salts are difficult to prepare, and there is no need to collect the growth rate data of large crystals because of a lack in application. The growth rate of a small crystal is usually lower than that of a large crystal [9]. 
Therefore, it is not adequate to use the growth rates of small crystals for the design of a pellet reactor.

The seeded technique for studying the crystal growth rate of readily soluble system looks straightforward as compared with that of sparingly soluble system. It seems that there are many problems to encounter in studying the latter system, including at least the following: the preparation of large seed crystals, the determination of supersaturation, the identification of metastable region, and the control of $\mathrm{pH}$ and supersaturation of solution. The large seed crystals of sparingly soluble salts seem difficult to prepare; however, this problem can be solved by using the gel growth method, which is a well-known technique to grow high-quality single crystals at a low cost [10]. The expression of supersaturation is different between the readily and sparingly soluble systems. The definition of relative supersaturation proposed by Nielsen and Toft [11] is adopted by many researchers. The concentrations of species, which are required to calculate ionic activity and thus supersaturation, were determined by using a computer program based on the algorithm of successive approximation of the ionic strength [12]. To study crystal growth, the solution supersaturation should be controlled within the metastable region; otherwise, nucleation would occur at higher supersaturation and mess up the operation. Although the metastable region of $\mathrm{CaCO}_{3}$ is not reported in the literature, experimental techniques are available [4] and experiments can be conducted to locate the region. During a crystal growth process, the solution $\mathrm{pH}$ will change, especially for the system that the species will form complex with hydrogen ion such as $\mathrm{CaCO}_{3}-\mathrm{H}_{2} \mathrm{O}$ system, and the supersaturation will shift either to a low value where crystal growth rate is negligible or to a high value where nucleation occurs. Therefore, the control of $\mathrm{pH}$ is essential and can be achieved by using an autotitrator [7,8].

In our laboratory, the growth kinetics of calcite crystals was studied in batch, pH-stat crystallizers of a stirred tank and fluidized-bed type. This report summarizes the experimental study regarding crystal growth rates of calcite in laboratory-scale equipments. After the seed crystals were prepared and the metastable region was located, the crystal growth data were evaluated from the consumption rate of titrant or calcium ion using an autotitrator. The factors that affect the crystal growth rates were investigated. Then the growth rate data were analyzed by the two-step growth model, thus the mass-transfer and surface-reaction coefficients were determined. Finally, the crystal growth kinetics of sparingly soluble systems was compared with that of readily soluble systems in the two types of crystallizer. This kind of comparison is worthwhile from the academic point of view. In the past, experiments of the two systems were conducted by different research groups, probably due to different experimental techniques involved.

The two-step growth model used in the analysis of crystal growth rate data takes account of the mass-transfer resistance and surface-reaction resistance in series and neglects all other resistances in a crystal growth process, which may be expressed mathematically by the following equations:

$G=K_{\mathrm{d}}\left(\sigma-\sigma_{\mathrm{i}}\right)=K_{\mathrm{do}} L^{a}\left(\sigma-\sigma_{\mathrm{i}}\right) \quad$ Bulk transport

$G=K_{\mathrm{r}} \sigma_{\mathrm{i}}^{r}=K_{\mathrm{ro}} L^{b} \sigma_{\mathrm{i}}^{r} \quad$ Surface reaction

where $G$ is the linear crystal growth rate, $\sigma$ the overall supersaturation, $\sigma_{\mathrm{i}}$ the interfacial supersaturation, $L$ the crystal size, $r$ the surface-reaction order, and $K_{\text {do }}$ and $K_{\text {ro }}$, the mass-transfer and surface-reaction coefficients, respectively.

\section{Determination of relative supersaturation}

The relative supersaturation of a readily soluble salt is usually expressed by the difference between solution concentration and saturation concentration divided by saturation concentration. This simplified expression of driving force is difficult to apply to sparingly soluble system because the species of crystallizing compound are usually not in equal concentration. In the literature, different expressions have been used. The relative supersaturation proposed by Nielsen and Toft [11] is adopted here:

$\sigma=\left(K_{\mathrm{IP}}^{1 / 2}-K_{\mathrm{sp}}^{1 / 2}\right) / K_{\mathrm{sp}}^{1 / 2}$

where $K_{\mathrm{IP}}$ is the ionic product, defined as $K_{\mathrm{IP}}=$ $a_{\mathrm{Ca}^{2+}} a_{\mathrm{CO}_{3}^{2-}}$, and $K_{\mathrm{sp}}$ is the solubility product of $\mathrm{CaCO}_{3}$. The activity of species, $a_{\mathrm{Ca}^{2+}}$ or $a_{\mathrm{CO}_{3}^{2-}}$, is the product of activity coefficient and ionic concentration of the respective species. The concentrations of ionic species are computed from the measured $\mathrm{pH}$, total calcium and carbonate concentration by successive approximation for the ionic strength $[12,13]$. The computer program contains the mass action and mass balance equations and the modified Debye-Hückle equation for calculating the activity coefficient [14]. In the operation of crystal growth experiment, supersaturation should be controlled in a suitable range; a low supersaturation gives a growth rate that is insignificant, and a high supersaturation causes nucleation that messes up the operation. The supersaturation is significantly affected by the solution $\mathrm{pH}$ because the concentration of $\mathrm{CO}_{3}^{2-}$ is a function of $\mathrm{pH}$ at a fixed concentration of total carbonate. As judged from the distribution of the total carbonate ions, existing as $\mathrm{CO}_{3}^{2-}, \mathrm{HCO}_{3}^{-}$and $\mathrm{H}_{2} \mathrm{CO}_{3}$, the suitable operation range of $\mathrm{CaCO}_{3}$ system is roughly between $\mathrm{pH} 8.5$ and 10.5.

\section{Experimental}

\subsection{Identification of metastable region}

To suppress nucleation in a crystal growth experiment, supersaturation should be kept in the metastable region, 
which is the area between supersolubility and solubility curves in a precipitation diagram [4]. The experimental procedures to identify the metastable region of $\mathrm{CaCl}_{2}-$ $\mathrm{Na}_{2} \mathrm{CO}_{3}-\mathrm{H}_{2} \mathrm{O}$ system are described elsewhere [15]. Briefly speaking, the supersolubility curve of $\mathrm{CaCO}_{3}$ is the boundary where nucleation occurs at a specified induction period for different species concentrations, i.e. $\mathrm{CaCl}_{2}$ and $\mathrm{Na}_{2} \mathrm{CO}_{3}$ solution of known concentrations are mixed and stirred for a period of time at which the solution becomes turbid. The metastable region of calcium carbonate is shown in Fig. 1 as $\mathrm{p} a_{\mathrm{Ca}^{2+}}$ versus $\mathrm{p} a_{\mathrm{CO}_{3}^{2-}}$, where $a_{\mathrm{Ca}^{2+}}$ and $a_{\mathrm{CO}_{3}^{2-}}$ are the activities of $\mathrm{Ca}^{2+}$ and $\mathrm{CO}_{3}^{2-}$, respectively. The activities are calculated from the concentrations by the same computer program for estimating supersaturation. The solubility curve is plotted in Fig. 1 using the solubility product of $\mathrm{CaCO}_{3}, \quad K_{\mathrm{sp}}=4.872 \times 10^{-9}$ [16]. The two curves are almost straight and parallel in the concentration range studied, and they are independent of $\mathrm{pH}$ values between 8.5 and 10.5 . The metastable zone width so determined is based on primary nucleation. In the experiments, the operation range was kept within one-half of the width to suppress the primary and secondary nucleation.

\subsection{Preparation of seed crystals}

Seed crystals used in the experiment were from two sources. The large sizes greater than $50 \mu \mathrm{m}$ were prepared by the gel growth technique or by curing natural calcite, and smaller sizes were obtained by curing the product from Nacalai Tseque. The size of large crystals was determined by sieving, i.e. the mean size of two close-cut sieves, and the size of smaller crystals was converted from the surface area measured by a BET surface area analyzer (Microneritics, $2100 \mathrm{D}$ ), taking the crystals as rhombohedron identified by SEM photographs. The identification of calcite was accomplished by using an IR spectra and X-ray powder diffraction pattern.

\subsection{Crystallization apparatus}

The crystallization system that contains a $\mathrm{pH}$-stat fluidized-bed crystallizer, a storage tank, and a pH-control system is shown in Fig. 2. In the study of crystal growth in a stirred tank, the fluidized bed is removed and the storage tank is used as the crystallizer. The key component of the set-up is an autotitrator or pH-stat apparatus (Kyoto Electronic, AT 200) that maintains the $\mathrm{pH}$ constant during an experiment. The experimental procedures are described in detail by Tai et al. $[13,15]$. In the reports, the procedures for estimating the linear crystal growth rates are also given. Briefly speaking, in the stirred-tank experiment, $\mathrm{CaCl}_{2}$ is in excess and the control of $\mathrm{pH}$ is done by adding $\mathrm{Na}_{2} \mathrm{CO}_{3}$ solution, thus the crystal growth rate is estimated

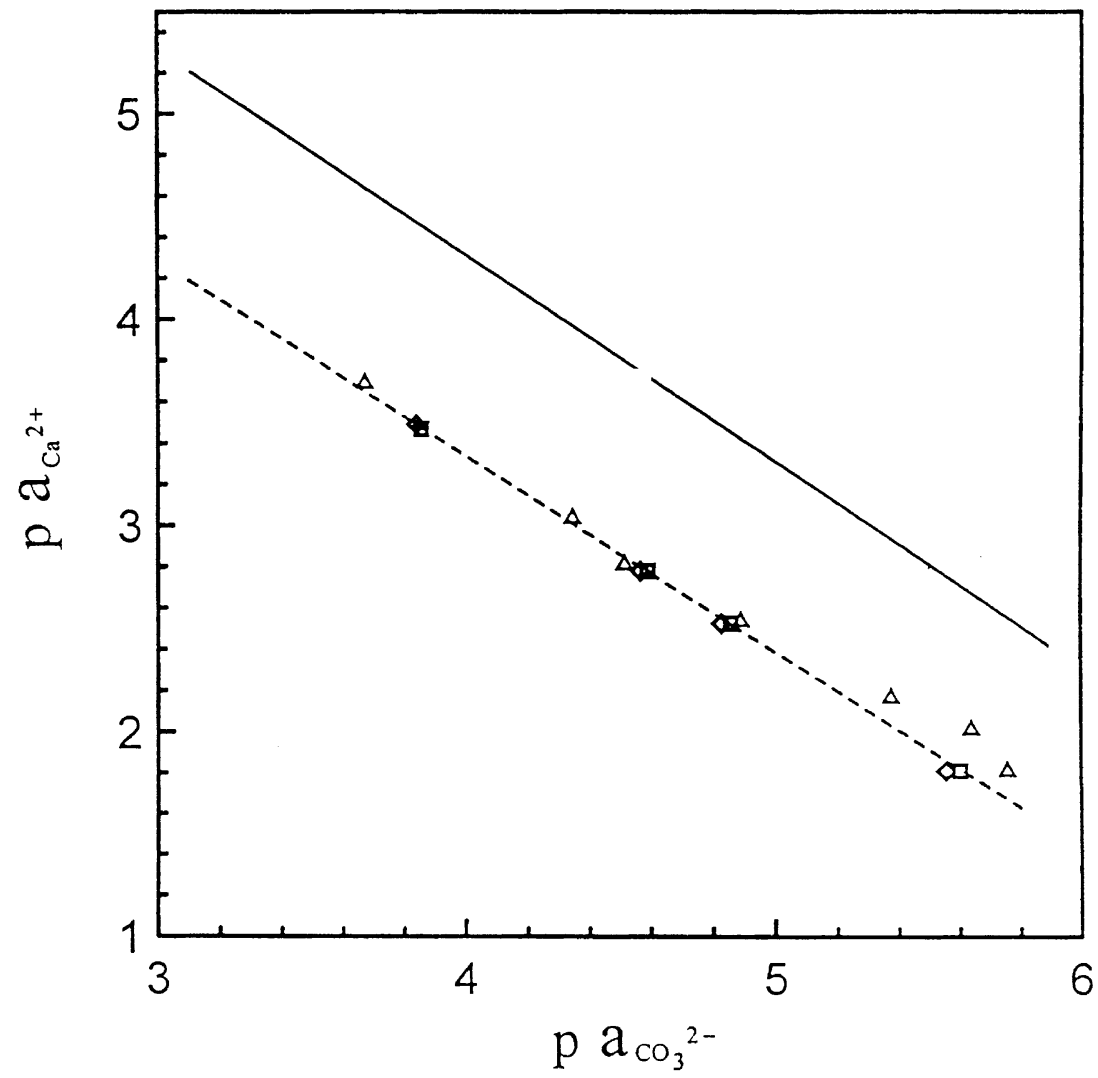

Fig. 1. Metastable region of calcium carbonate: $a_{\mathrm{Ca}^{2+}}$ vs. $\mathrm{p} a_{\mathrm{CO}_{3}^{2-}} .-$ Solubility curve; -- supersolubility curve; $\Delta \mathrm{pH}=8.5, \diamond \mathrm{pH}=9.5$, $\square \mathrm{pH}=10.5$. 


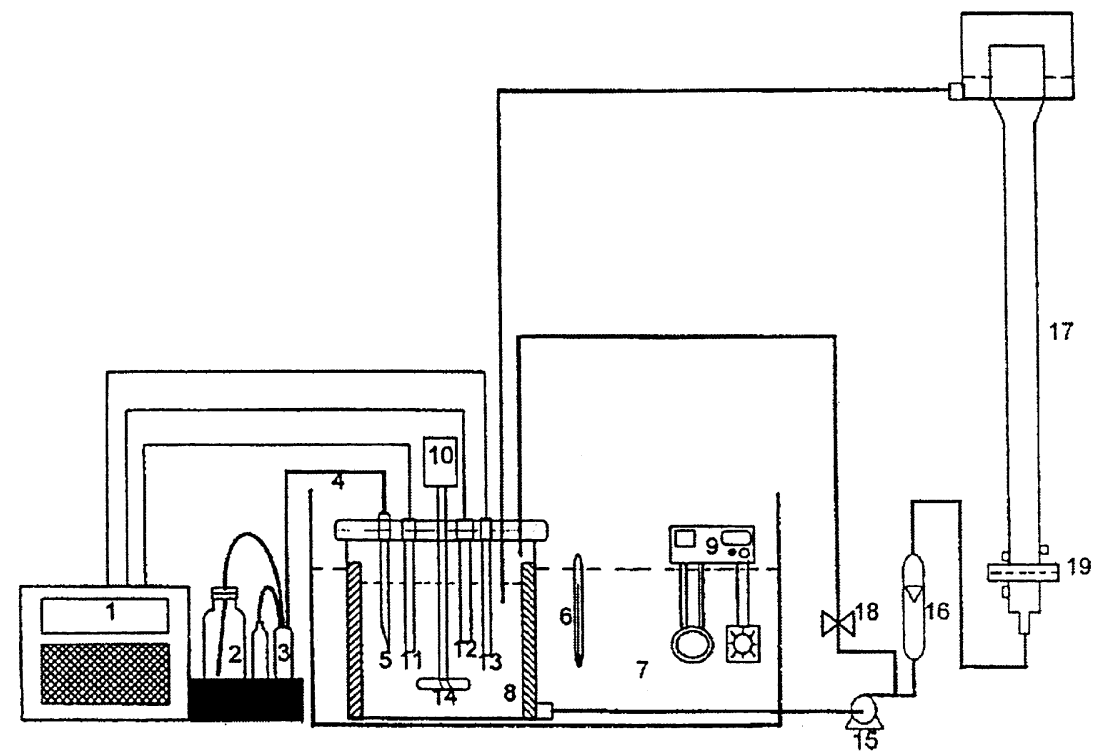

Fig. 2. A pH-stat crystallization system. (1) pH and temperature indicator, (2) reagent bottle, (3) pumping system of reagent, (4) reagent delivering line, (5) burette, (6) thermometer, (7) water bath, (8) storage tank, (9) temperature controller, (10) motor, (11) glass electrode, (12) reference electrode, (13) thermocompensator, (14) axial-flow impeller, (15) magnetic motor, (16) flowmeter, (17) fluidized-bed crystallizer, (18) recycle valve, (19) distributor.

by the consumption rate of the titrant; in the fluidized-bed experiment, the $\mathrm{pH}$ is controlled by adding $\mathrm{NaOH}$ solution and the crystal growth rate is evaluated by the consumption rate of calcium ion.

\section{Crystal growth kinetics in a stirred tank}

The working equation for estimating the parameters of the two-step growth model is derived by combing Eqs. (1) and (2).

$$
\frac{G}{K_{\mathrm{do}} L^{a}}+\left(\frac{G}{K_{\mathrm{ro}} L^{b}}\right)^{1 / r}=\sigma
$$

When experimental data of all crystal sizes were used in a regression analysis, they did not fit well with Eq. (4). Therefore, growth rate data of small crystals for 2, 5, and 8 $\mu \mathrm{m}$, and large crystals between 58 and $230 \mu \mathrm{m}$ were analyzed separately. The results are shown below:

$$
\begin{aligned}
& G=1.999 \times 10^{-9} L^{-0.00256}\left(\sigma-\sigma_{\mathrm{i}}\right) \\
& G=1.345 \times 10^{-11} L^{0.627} \sigma_{\mathrm{i}}^{2.20}
\end{aligned}
$$

for small crystals, where $G$ is in $\mathrm{m} / \mathrm{s}, L$ in $\mu \mathrm{m}$, and $\sigma$ and $\sigma_{\mathrm{i}}$ are dimensionless. In addition,

$$
\begin{aligned}
& G=5.887 \times 10^{-10} L^{0.434}\left(\sigma-\sigma_{\mathrm{i}}\right) \\
& G=1.292 \times 10^{-11} L^{0.604} \sigma_{\mathrm{i}}{ }^{2.02}
\end{aligned}
$$

for large crystals.

It is concluded that the mass-transfer rate is independent of crystal size for small crystals but increases with increas- ing size for large crystals. This is because the exponent of $L$ is a very small number in Eq. (5) and a significant positive figure in Eq. (7). On the other hand, the surfacereaction rate is similar for the whole size range by comparing Eqs. (6) and (8) and increases with an increase in crystal size.

Once the parameters in the two-step growth model are available, a judgement on the importance of each step can be made using the concept of effectiveness factor [17]. The

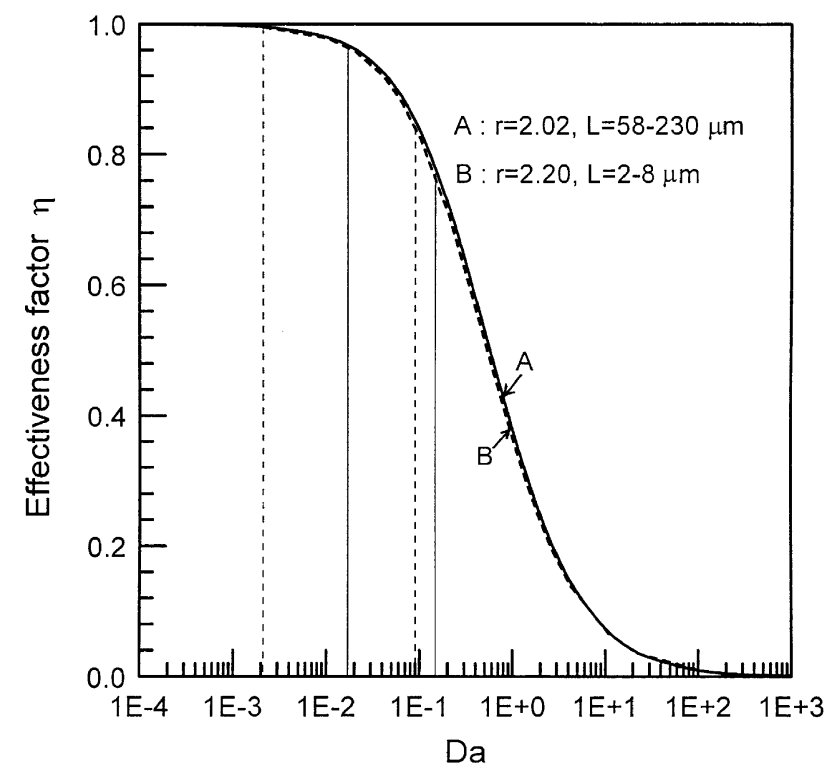

Fig. 3. Operating range of Damköhler number and corresponding effectiveness factor for the $\mathrm{CaCO}_{3}$ system. 


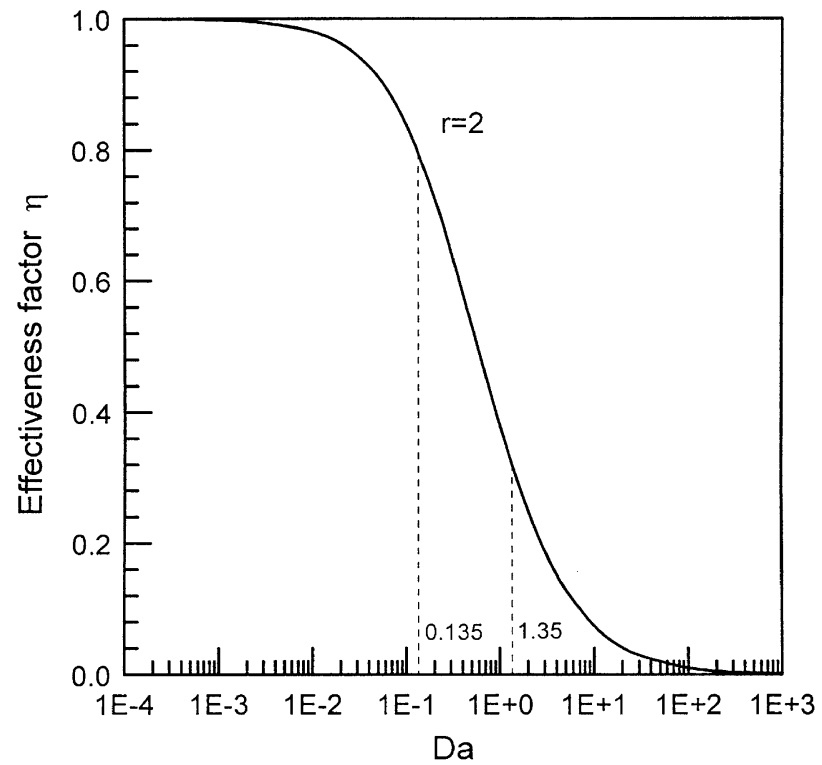

Fig. 4. Operating range of Damköhler number and corresponding effectiveness factor for potassium alum system.

surface-reaction effectiveness factor $\eta$ for an $r$ th-order rate is:

$\eta=(1-\eta D a)^{r}$

where $D a$ is expressed by:

$D a=K_{\mathrm{ro}} L^{b-a} \sigma^{r-1} / K_{\mathrm{do}}$

When $\eta$ approaches 1 , the crystal growth process is surface-reaction controlled; on the other hand, the growth process is mass-transfer controlled when $\eta$ approaches zero. Eq. (9) is plotted in Fig. 3, which shows that the mass-transfer resistance and surface-reaction resistance are both significant for large crystals, and the mass-transfer resistance becomes less important at lower supersaturation for smaller crystals. The result of large crystals is consistent with that of one of the readily soluble salts, potassium alum, as shown in Fig. 4 for particle size being between 100 and $700 \mu \mathrm{m}$ [19]. On the other hand, the result of small crystals approaches the conclusion made by Garside and Jančić [18] that the growth process of potassium alum, a readily soluble salt, is controlled by the surface reaction for micron-sized particles.
The parameters in the two-step growth model of the sparingly soluble salt $\left(\mathrm{CaCO}_{3}\right)$ are compared with those of readily soluble salts as shown in Table 1 [13]. The variation in parameters can be understood because various types of crystals are included in the table, i.e. readily soluble inorganic (potassium sulfate, potassium alum), readily soluble organic (succinic acid), and sparingly soluble inorganic (calcite). The kinetic behaviors of large inorganic crystals of readily soluble and sparingly soluble salts are similar, i.e. mass-transfer and surface-reaction coefficients increase with increasing crystal size. However, the organic crystal (succinic acid) behaves differently in the crystal size effect on mass-transfer coefficient, which decreases with an increase in crystal size. There is no explanation for this type of growth kinetics at the present time. For small crystals, no comparison can be made because the parameters of the two-step model are not available for the readily soluble crystals.

The findings on the mass-transfer rates of various crystal sizes are consistent with the growth behaviors inferred by Mullin [9]: large particles have higher terminal velocity and, in cases where diffusion plays a significant role in the growth process, the larger the crystals, the higher the growth rate; for crystals smaller than about $10 \mu \mathrm{m}$, their sizes are smaller than that of turbulent eddies and they may be growing in a virtually stagnant medium even in a well-agitated system, thus the mass-transfer coefficient is independent of size. As to the surface-reaction rate, the size effect has been postulated by Garside and Davey [22]. The larger the crystals, the more energetically will they collide in agitated suspensions and the greater is the potential for surface damage, or the number of dislocations in a crystal increases with size due to mechanical stresses [9]. Both of these effects favor faster surface-reaction kinetics and lead to higher growth rates with increasing crystal size.

\section{Crystal growth kinetics in a fluidized bed}

Depending on the superficial velocity applied in an operation, two types of fluidized bed are classified, i.e. the dense bed and the lean bed. The former is conducted at a

Table 1

Parameters of the two-step growth model for calcite and other substances grown in an agitated tank: mass-transfer coefficient, $K_{\mathrm{d}}=K_{\mathrm{do}} L^{a}$; surface-integration coefficient, $K_{\mathrm{r}}=K_{\mathrm{ro}} L^{b}$

\begin{tabular}{|c|c|c|c|c|c|c|}
\hline Author & Systems & $\begin{array}{l}\text { Agitation } \\
\text { speed (rpm) }\end{array}$ & $\begin{array}{l}\text { Seed size } \\
(\mu \mathrm{m})\end{array}$ & $a$ & $b$ & $\mathrm{r}$ \\
\hline Garside et al. (1974) [19] & Potassium sulfate & $500-800$ & $570-1180$ & 0.130 & 0.700 & 2.40 \\
\hline Tai and Yu (1989) [20] & Potassium alum & 950 & $100-700$ & 0.581 & 0.583 & 2.00 \\
\hline Qiu and Rasmuson (1990) [21] & Succinic acid & $355-800$ & $400-710$ & -0.170 & 1.600 & 3.00 \\
\hline \multirow[t]{2}{*}{ Tai et al. (1993) [13] } & Calcite & 800 & $58-230$ & 0.434 & 0.604 & 2.02 \\
\hline & & & $2-8$ & -0.0256 & 0.627 & 2.20 \\
\hline
\end{tabular}


superficial velocity lower than the particle terminal velocity to give the bed a clear solution-suspension boundary; in the latter, the superficial velocity is approaching the terminal velocity and the particles may move freely in the bed with no boundary. In the operation of a pellet reactor, a dense bed is more advantageous as far as seed loading is concerned. Therefore, factors that affect the calcite growth rate in a dense bed were investigated, including superficial velocity, particle size, supersaturation, $\mathrm{pH}$, and ionic strength.

When keeping the ionic strength and $\mathrm{pH}$ constant, the crystal growth rate increases with an increase in crystal size ranging between 460 and $920 \mu \mathrm{m}$; on the other hand, the growth rates of $460-\mu \mathrm{m}$ seed are rather constant at various superficial velocities of $1.42,2.36,3.54$ and 4.73 $\mathrm{m} / \mathrm{s}$ [15], in spite of a great change in bed voidage and bed expansion. The mechanisms of crystal growth related to crystal size and superficial velocity will be clarified after the growth rate data are analyzed by the two-step growth model.

According to different sources, the surface-reaction order of calcite growth is approximately $2[6,8,13]$. Taking $r=2$, Eqs. (2) and (3) are combined to give:

$\frac{\sigma}{\sqrt{G}}=\frac{1}{K_{\mathrm{d}}} \sqrt{G}+\frac{1}{\sqrt{K_{\mathrm{r}}}}$,

thus the mass-transfer and surface-reaction coefficients can be evaluated by plotting $\sigma / \sqrt{ } G$ versus $\sqrt{ } G$. The obtained $K_{\mathrm{d}}$ and $K_{\mathrm{r}}$ are listed in Table 2. Despite the variation in particle size and superficial velocity, the values of $K_{\mathrm{d}}$ are rather constant. The largest deviation from the average value of $K_{\mathrm{d}}, 1.32 \times 10^{-2}$, is about $15 \%$. The surface-reaction coefficient $K_{\mathrm{r}}$ increases with an increase in crystal size when we compare the first three runs in Table 2, and $K_{\mathrm{r}}$ is independent of superficial velocity as shown by the last three runs. The dependence of $K_{\mathrm{r}}$ on crystal size is similar to the readily soluble systems $[23,24]$ and has been explained in the Section 4. The independence of $K_{\mathrm{r}}$ on superficial velocity is easily understood. Because surface reaction is an interfacial phenomenon, it should not be disturbed by liquid flow under a mild agitation, such as in

Table 2

Mass-transfer and surface-reaction coefficients of calcite crystal estimated by using Eq. (11) at $\mathrm{pH}=9.5$ and $I=0.0025 \mathrm{kmol} / \mathrm{m}^{3}$

\begin{tabular}{lllll}
\hline Run no. & $\begin{array}{l}\text { Particle } \\
\text { size } \\
(\mu \mathrm{m})\end{array}$ & $\begin{array}{l}\text { Superficial } \\
\text { velocity } \\
(\mathrm{m} / \mathrm{s})\end{array}$ & $\begin{array}{l}K_{\mathrm{d}} \\
\left(10^{-10} \mathrm{~m} / \mathrm{s}\right)\end{array}$ & $\begin{array}{l}K_{\mathrm{r}} \\
\left(10^{-9} \mathrm{~m} / \mathrm{s}\right)\end{array}$ \\
\hline A-4-1-O20 & 920 & 3.54 & 1.45 & 13.78 \\
A-4-2-O13 & 650 & 3.54 & 1.42 & 2.25 \\
A-2-2-M14 & 460 & 3.54 & 1.33 & 0.30 \\
A-1-4-M08, 16 & 460 & 2.36 & 1.11 & 0.31 \\
A-2-4-M12, 13 & 460 & 1.42 & 1.29 & 0.34 \\
\hline
\end{tabular}

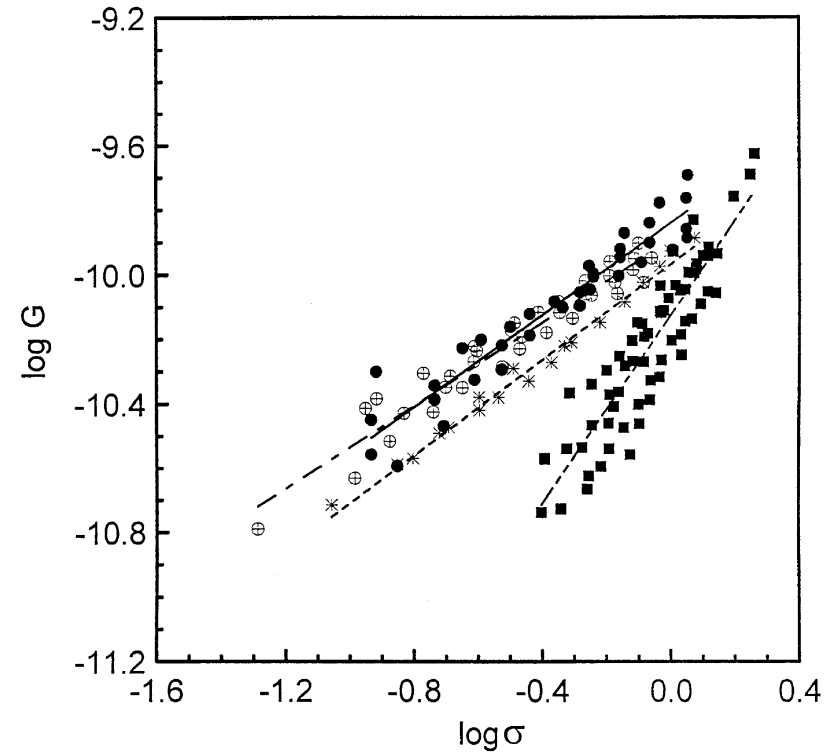

Fig. 5. $\log G$ vs. $\log \sigma$ showing effect of ionic strength on the growth rate of $\mathrm{CaCO}_{3}$ crystal at $L=460 \mu \mathrm{m} ; \mathrm{pH}=9.5 ; T=25^{\circ} \mathrm{C}$. Symbols and corresponding ionic strength $\left(\mathrm{kmol} / \mathrm{m}^{3}\right): \oplus 0.0340 ; 0.0185 ;{ }^{*} 0.0105$; - 0.0025 .

a fluidized bed. To explain the constant $K_{\mathrm{d}}$, an argument based on the relative velocity between solution and particle is presented here. As the superficial velocity is reduced, the bed voidage and thus the cross-sectional area for the passage of solution becomes smaller. As a result, the relative velocity, which determines the mass-transfer coefficient, does not vary much for different crystal sizes and superficial velocities. Besides, we can arrive at the constant $K_{\mathrm{d}}$ using a correlation proposed by Kunii and Levenspiel [25]. The argument is presented somewhere else [15]. The variation of $K_{\mathrm{d}}$ with particle size or superficial velocity is actually less than $15 \%$. This result is similar to that reported by Budz et al. [23] in Table 3 of their paper for the $\mathrm{K}$-alum system, in which the highest deviation from the average value is about $10 \%$. The constant masstransfer coefficient is also reported by Tournie et al. [26] for crystal dissolution process, after they analyzed extensive dissolution data of both lean and dense fluidized bed in the literature.

Once $K_{\mathrm{d}}$ and $K_{\mathrm{r}}$ are obtained, the effects of crystal size and superficial velocity on the kinetic behaviors of $\mathrm{CaCO}_{3}$ growth can be explained in terms of the two-step growth model. The surface-reaction step is responsible for the size-dependent growth because $K_{\mathrm{r}}$ increases with increasing crystal size and $K_{\mathrm{d}}$ is independent of crystal size. On the other hand, the crystal growth rates of the same size are constant at various superficial velocities because $K_{\mathrm{d}}$ and $K_{\mathrm{r}}$ are independent of superficial velocity.

The effects of supersaturation and ionic strength on calcite growth rate are shown in Fig. 5. The ionic strength varying from 0.0025 to $0.0340 \mathrm{kmol} / \mathrm{m}^{3}$ was adjusted by 
adding $\mathrm{NaCl}$ solution. For all levels of ionic strength, the growth rates of calcite increase with an increase in supersaturation. At the same supersaturation, the growth rates increase with ionic strength from 0.0025 to 0.0185 $\mathrm{kmol} / \mathrm{m}^{3}$; however, a further increase in ionic strength is no more effective. The slope of $\log G-\log \sigma$ curve changes at the lowest ionic strength, indicating a change in the weighting of various resistances to crystal growth. The kinetic behavior of $\mathrm{PbF}_{2}$ crystal reported by Stubicar et al. [27] is similar to that of $\mathrm{CaCO}_{3}$ as far as the effect of ionic strength is concerned.

Fig. 6 shows the effects of $\mathrm{pH}$ at two levels, 8.5 and 9.5 , on the growth rate of calcite. Similar to the effects of ionic strength, different slopes of $\log G-\log \sigma$ plot are obtained at different $\mathrm{pH}$, and the influence of $\mathrm{pH}$ becomes insignificant at higher supersaturation. Stubicar et al. [27] reported that the crystal growth rate of $\alpha$-fluoride increased with $\mathrm{pH}$ when the solution $\mathrm{pH}$ was lower than the isoelectric point at $\mathrm{pH}$ 5.6. The results obtained in this experiment of calcite is consistent with $\alpha$-lead fluoride because the isoelectric point of calcite is between 9 and 10 [28]. Hohl et al. [29] also demonstrated that the $\mathrm{CaHPO}_{4}$. $2 \mathrm{H}_{2} \mathrm{O}$ and hydroxyapatite crystals grew more slowly at $\mathrm{pH}$ values below, than they do above, the isoelectric point. Besides, the $\mathrm{pH}$ effect for $\mathrm{CaF}_{2}$ growth is reported by Tai et al. [30] as shown in Fig. 7. The pH effect implies that the charge on the crystal face is important in the building of growth units into the lattice [4]. The effects of ionic strength and $\mathrm{pH}$ are seldom reported for readily soluble salts; however, they are significant to the sparingly soluble salts. This is probably because the ionic strength is already very high in a supersaturated solution of readily soluble

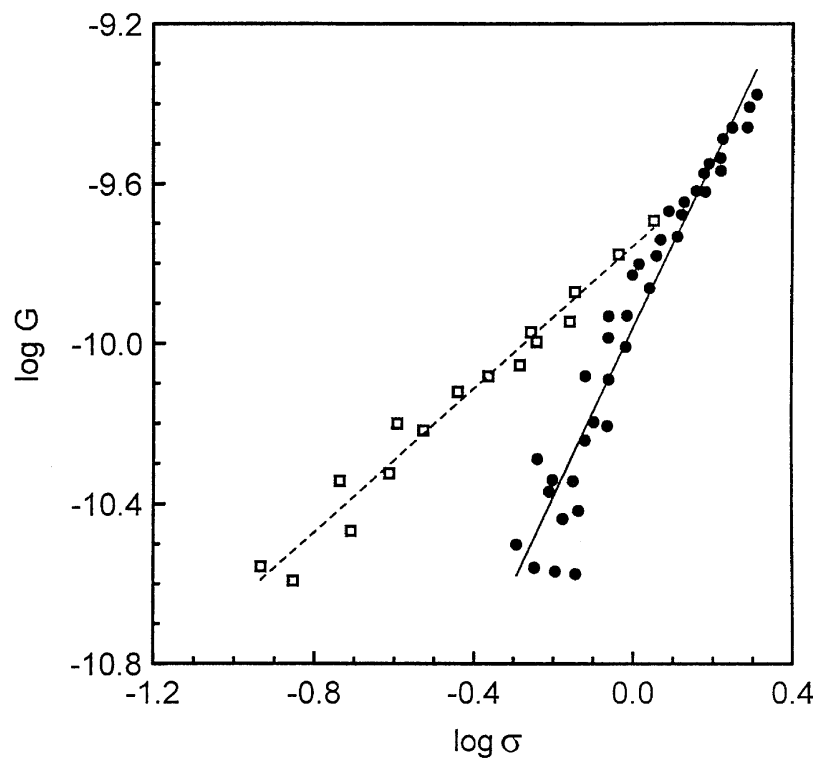

Fig. 6. $\log G$ vs. $\log \sigma$ showing crystal growth rate of $\mathrm{CaCO}_{3}$ at two $\mathrm{pH}$ values: $I=0.018 \mathrm{kmol} / \mathrm{m}^{3} ; L=460 \mu \mathrm{m} ; T=25^{\circ} \mathrm{C} ; u=2.36 \times 10^{-2}$ $\mathrm{m} / \mathrm{s} ; \mathrm{pH}=8.5, \square \mathrm{pH}=9.5$.

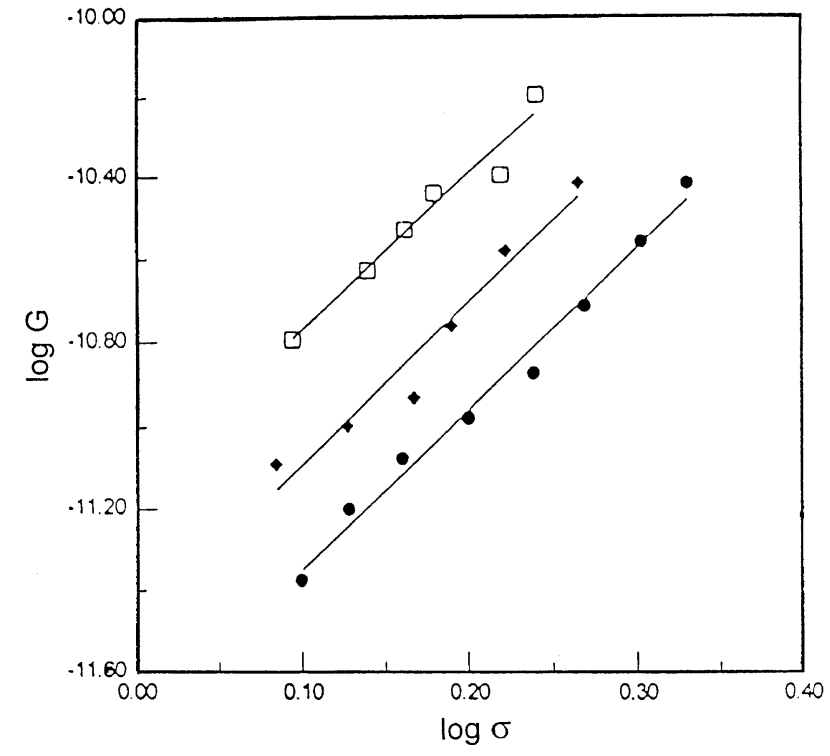

Fig. 7. $\mathrm{pH}$ effect on the crystal growth of $\mathrm{CaF}_{2}$, using $388-\mu \mathrm{m}$ fluoride crystal seed. $I=0.11 \mathrm{kmol} / \mathrm{m}^{3} ; u=2.4 \times 10^{-2} \mathrm{~m} / \mathrm{s} ; \square \mathrm{pH}=10, \diamond$ $\mathrm{pH}=9, \bigcirc \mathrm{pH}=8$.

systems, and the $\mathrm{pH}$ does not change during the solid formation.

\section{Concluding remarks}

The pH-stat apparatus is suitable for studying crystal growth kinetics of sparingly soluble salts. Growth experiments should be conducted in the metastable region that lies between two parallel straight lines, i.e. solubility curve and supersolubility curve, when the precipitation diagram is constructed by plotting $\mathrm{p} a_{\mathrm{Ca}^{2+}}$ versus $\mathrm{p}_{\mathrm{CO}_{3}^{2-}}$. Factors that affect the calcite growth rates are identified, including supersaturation, superficial velocity, particle size, $\mathrm{pH}$, and ionic strength. Then the two-step crystal growth model is employed to analyze the calcite growth data of constant $\mathrm{pH}$ and ionic strength and much useful kinetic information is revealed. The obtained $K_{\mathrm{d}}$ and $K_{\mathrm{r}}$, mass-transfer and surface-reaction coefficients, can be used to explain the crystal growth behaviors. In a stirred tank operated at fixed agitation rate, both coefficients are size-dependent except for the mass-transfer of small crystals $(<10 \mu \mathrm{m})$, and the surface reaction is responsible for the size-dependent growth of small crystals. In a fluidized bed operated in a dense mode, the mass-transfer coefficient is independent of crystal size and superficial velocity and the surface-reaction coefficient is size-dependent but not influenced by superficial velocity; thus the superficial velocity has little effect on the growth rates of calcite of the same size and the surface reaction is responsible for the size effect. The crystal growth behaviors of this sparingly soluble salt are similar to that of readily soluble salts. For the sparingly soluble systems, two more variables, i.e. $\mathrm{pH}$ and ionic 
strength, appear important. This finding together with the similar results previously reported imply that the charge on crystal face is important in the building of growth units into the crystal lattice.

\section{Notations}

a Exponent of crystal size in Eq. (1)

$a_{\mathrm{Ca}^{2+}} \quad$ Activity of calcium ion $\left(\mathrm{kmol} / \mathrm{m}^{2}\right)$

$a_{\mathrm{CO}_{3}^{2-}} \quad$ Activity of carbonate ion $\left(\mathrm{kmol} / \mathrm{m}^{2}\right)$

$b \quad$ Exponent of crystal size in Eq. (2)

Da Damköhler number

$G \quad$ Linear crystal growth rate $(\mathrm{m} / \mathrm{s})$

I Ionic strength $\left(\mathrm{kmol} / \mathrm{m}^{3}\right)$

$K_{\mathrm{d}} \quad$ Mass-transfer coefficient $(\mathrm{m} / \mathrm{s})$

$K_{\text {do }} \quad$ Size-independent mass-transfer coefficient $(\mathrm{m} / \mathrm{s})$

$K_{\mathrm{IP}} \quad$ Ionic product $\left(\mathrm{kmol} / \mathrm{m}^{3}\right)^{2}$

$K_{\mathrm{r}} \quad$ Surface-reaction coefficient $(\mathrm{m} / \mathrm{s})$

$K_{\text {ro }} \quad$ Size-independent mass-transfer coefficient $(\mathrm{m} / \mathrm{s})$

$K_{\text {sp }} \quad$ Solubility product $\left(\mathrm{kmol} / \mathrm{m}^{3}\right)^{2}$

$L \quad$ Crystal size (in $\mathrm{m}$ or $\mu \mathrm{m}$ as specified in the text)

$r \quad$ Surface-reaction order

$T \quad$ Temperature $\left({ }^{\circ} \mathrm{C}\right)$

$\eta \quad$ Effectiveness factor of crystal growth

$\sigma \quad$ Bulk relative supersaturation

$\sigma_{\mathrm{i}} \quad$ Interfacial relative supersaturation

\section{Acknowledgements}

The authors gratefully acknowledge the financial support of the National Science Council of the Republic of China through the years in the area of crystal growth.

\section{References}

[1] J.C. van Dijk, D.A. Wilms, J. Water SRT-Aqua. 40 (1991) 263-280.

[2] M.M. Seckler, O.S.L. Bruinsma, G.M. van Rosmalen, J.C. van Dijk, F. Delgorge, in: A. Mersmann (Ed.), Industrial Crystallization, vol. 90, Garmisch-Parten Kirchen, FRG, 1990, pp. 134-148.
[3] P. Dirken, E. Baars, A. Graveland, F.C. Woensdregt, in: A. Mersmann (Ed.), Industrial Crystallization, vol. 90, Garmisch-Parten Kirchen, FRG, 1990, pp. 95-100.

[4] O. Söhnel, J. Garside, Precipitation-Basic Principles and Industrial Applications. Butterworth-Heinemann, Boston, 1992, p. 149.

[5] D.M. Killer, R.E. Massey, O.E. Hileman, Can. J. Chem. 56 (1978) 831-838.

[6] G.H. Nancollas, M.M. Reddy, J. Colloid Interface Sci. 37 (1971) $824-830$

[7] E.K. Giannimaras, P.G. Koutsoukos, J. Colloid Interface Sci. 116 (1987) 423-430.

[8] J. Christoffersen, M.R. Christoffersen, J. Cryst. Growth 100 (1990) 203-211.

[9] J.W. Mullin, Crystallization. 3rd edn., Butterworth-Heinemann, Oxford, 1993, pp. 237-238.

[10] H.K. Henisch, Crystals in Gels and Liesegang Rings. 2nd edn., Cambridge Univ. Press, Cambridge, 1988, p. 48.

[11] A.E. Nielsen, J.M. Toft, J. Cryst. Growth 67 (1984) 278-288.

[12] G.H. Nancollas, Interactions in Electrolyte Solutions. Elsevier, Amsterdam, 1966, p. 85.

[13] C.Y. Tai, P.-C. Chen, S.-M. Shih, AIChE J. 39 (1993) 1472-1482.

[14] J.N. Butler, Ionic Equilibrium. Addison-Wesley, Reading, MA, 1964, p. 432.

[15] C.Y. Tai, W.-C Chien, C.Y. Chen, AIChE J. 45 (1999) 1605-1614.

[16] O. Söhnel, J. Garside, Precipitation-Basic Principles and Industrial Applications. Butterworth-Heinemann, Boston, 1992, p. 296.

[17] J. Garside, Chem. Eng. Sci. 26 (1971) 1425-1431.

[18] J. Garside, S.J. Jančić, AIChE J. 22 (1976) 887-894.

[19] J. Garside, J.W. Mullin, S.W. Das, Ind. Eng. Chem. Fundam. 13 (1974) 299-305.

[20] C.Y. Tai, K.H. Yu, J. Cryst. Growth 96 (1989) 849-855.

[21] Y. Qiu, C. Rasmuson, AIChE J. 36 (1990) 665-676.

[22] J. Garside, R.J. Davey, Chem. Eng. Commun. 4 (1980) 393-424.

[23] J. Budz, P.H. Karpinski, Z. Nuruc, AIChE J. 30 (1984) 710-717.

[24] C.Y. Tai, C.-Y. Chen, J.-F. Wu, Chem. Eng. Commun. 56 (1987) 329-340.

[25] D. Kunii, O. Levenspiel, Fluidization Engineering. Wiley, New York, 1969, p. 167.

[26] P. Tournie, C. Larguerie, J.P. Couderc, Chem. Eng. Sci. 34 (1979) $1247-1255$.

[27] N. Stubicar, B. Markoric, A. Tonejc, M. Stubicar, J. Cryst. Growth 130 (1993) 300-304.

[28] J.S. Reed, Introduction to the Principles of Ceramic Processing. Wiley, Singapore, 1989, p. 134.

[29] H. Hohl, P.G. Koutsoukos, G.H. Nancollas, J. Cryst. Growth 57 (1982) 325-335.

[30] C.Y. Tai, T.M. Tsao, P.-C. Chen, M.S. Lee, Industrial Crystallization, vol. 96, Toulouse, France, 1996, pp. 123-128. 